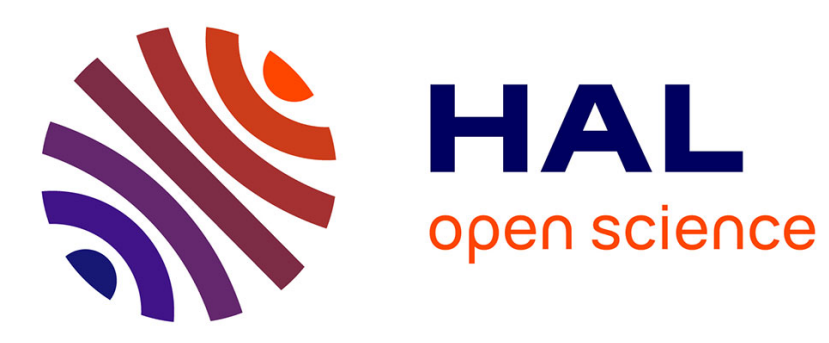

\title{
Interfacially driven transport theory: a way to unify Marangoni and Osmotic flows
}

Patrice Bacchin, Kirill Glavatskiy, Vincent Gerbaud

\section{To cite this version:}

Patrice Bacchin, Kirill Glavatskiy, Vincent Gerbaud. Interfacially driven transport theory: a way to unify Marangoni and Osmotic flows. Physical Chemistry Chemical Physics, 2019, pp.1-11. 10.1039/c9cp00999j . hal-02134681

\section{HAL Id: hal-02134681 \\ https://hal.science/hal-02134681}

Submitted on 20 May 2019

HAL is a multi-disciplinary open access archive for the deposit and dissemination of scientific research documents, whether they are published or not. The documents may come from teaching and research institutions in France or abroad, or from public or private research centers.
L'archive ouverte pluridisciplinaire HAL, est destinée au dépôt et à la diffusion de documents scientifiques de niveau recherche, publiés ou non, émanant des établissements d'enseignement et de recherche français ou étrangers, des laboratoires publics ou privés. 


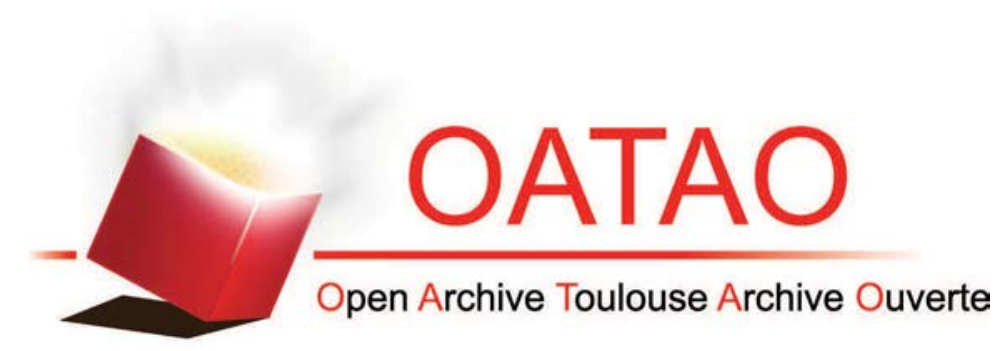

\section{Open Archive Toulouse Archive Ouverte (OATAO)}

OATAO is an open access repository that collects the work of some Toulouse researchers and makes it freely available over the web where possible.

This is an author's version published in: https://oatao.univ-toulouse.fr/23813

\section{Official URL :}

\section{To cite this version :}

Bacchin, Patrice $\fallingdotseq$ and Glavatskiy, Kirill and Gerbaud, Vincent $\leftrightarrows$ Interfacially driven transport theory: a way to unify Marangoni and Osmotic flows. (2019) Physical Chemistry Chemical Physics. 1-11. ISSN 1463-9076

Any correspondence concerning this service should be sent to the repository administrator: tech-oatao@listes-diff.inp-toulouse.fr 


\title{
Interfacially driven transport theory: a way to unify Marangoni and Osmotic flows
}

Author's version

Cite this:DOI: $10.1039 /$ c9cp00999j

\author{
Patrice Bacchin, ${ }^{*}$, Kirill S. Glavatskiy ${ }^{b}$, Vincent Gerbaud ${ }^{a}$
}

\begin{abstract}
We show that the solvent behaviour in both diffusio-osmosis and Marangoni flow can be derived from a simple model of colloid-interface interactions. We demonstrate that the direction of the flow is regulated by a single value of the attractive parameter covering the purely repulsive and attractive - repulsive interaction cases. The proposed universality between diffusio-osmosis and Marangoni flow is extended further to include diffusio-phoresis. In particular, an object immersed to a colloidal solution moves towards the low concentration of the colloidal particles in the case of colloid-interface repulsion and towards the high concentration of the colloidal particles in the case of colloid-interface attraction. The approach combines the methods of fluid dynamics, molecular physics and transport phenomena and provides a tractable explanation of how the colloid-interface interactions affect the momentum balance and the transport phenomena (interfacially driven transport).
\end{abstract}

\section{Introduction}

One of the distinct features of multiphase flow is presence of an effective interface, that sets a boundary between adjacent phases. In particular, an interface separates fluid droplets flowing within another fluid, or solid colloidal particles flowing in a solution. Alternatively, interfaces are present between the membrane and feed/permeate in filtration process, between gas and liquid in the distillation process, or between the fluid and the wall in microfluidic devices. It has been known for long that in equilibrium the interface has its own thermodynamic properties, which affects the state of the system ${ }^{1}$. In recent years it has been shown that this is also true in non-equilibrium conditions, which sets a new view point in a way that the properties of the interface may affect the flow ${ }^{2-4}$.

One of the key factors, which may influence the overall multiphase flow, is the variation of thermodynamic properties along the interface ${ }^{5,6}$. Even in the case of an isotropic fluid, which does not have a preferential direction on the molecular scale, the flow itself introduces anisotropy in the system ${ }^{7}$. In particular, the direction of the flow becomes the preferential direction for the fluid, so, on the molecular scale, fluid reveals some anisotropy. On the other hand, the presence of an interface breaks the fluid isotropy and one distinguishes two distinct directions along and perpendicular to the interface. Fluid flowing near the interface may induce some inhomogeneities in its properties, which feed back and modify the flow characteristics ${ }^{8,9}$.

\footnotetext{
a. Laboratoire de Génie Chimique, Université de Toulouse, CNRS, INP, UPS, Toulouse, France.patrice.bacchin@univ-tlse3.fr, vincent.gerbaud@ensiacet.fr

b. Centre for Complex Systems, The University of Sydney, NSW, Australia.

k.s.glavatskiy@gmail.com

*corresponding author: patrice.bacchin@univ-tlse3.fr.
}

It might be unusual to read in the title the terms "Marangoni flow", and "osmosis" in a single context along with the term "diffusiophoresis" in this paper, as each one occurs in a physically different environment. In particular, diffusiophoresis occurs in a bulk fluid, diffusio-osmosis occurs in the presence of a solid-fluid interface, while Marangoni flow occurs in the presence of a fluid-fluid interface. This must not confuse the reader, however, since the nature of the interface is not the main factor which results in the observed phenomena. What is more important is the presence of an interface together with the existence of a concentration gradient ${ }^{10}$.

In this spirit, it would be instructive to extend the meaning of these terms as follows. Diffusio-osmosis is the fluid flow along an interface in the direction of the concentration gradient, while Marangoni is the fluid flow along an interface in the direction opposite to the concentration gradient. Thus, one does not care about the nature of the concentration gradient (whether it is created by the variation of the surface tension as required for the standard Marangoni flow, or by the adsorption ability of the wall, as required by the standard diffusionosmosis), neither about the nature of the interface (whether it is solid-fluid or fluid-fluid). Such a coarse-graining view is legitimate, since there exists a direct analogy between the chemical interactions occurring in the corresponding systems ${ }^{10}$. One of the physical systems for which these phenomena may be of importance is a membrane. Membranes are used in various natural and industrial filtration processes ${ }^{11-14}$ and are typically viewed as series of interconnected pores, separated by solid walls. Alternatively, the fluid flowing through these pores can be viewed as the flow around solid objects, which perturb the homogeneous flow. For a regular membrane structure, one can identify a "unit cell" of solid objects, which is repeated several times. The classical flow pattern is determined by the particular structure of the object and is the solution of a fluid 
dynamics problem with corresponding boundary conditions. In membranes, fluid interactions with the membrane material are strong and have to be explicitly accounted. Microscopic details of colloidal interaction have been viewed important for the problems of colloidal diffusion in bulk fluids ${ }^{15-16}$ (in terms of interactions with solvent), confined geometries 17 (in terms of particle-wall interactions), and in the asymmetric environment 18. A recent development within the DLVO theory allows one to include these interactions explicitly in the context of fluid dynamics ${ }^{19}$, in particular to the flow through membranes.

Here we extend this approach to study interfacially driven transport. As we want to treat the interactions between a fluid and objects explicitly, we deal essentially with a multiphase flow, having the interface between the fluid and the objects. The fluid is a two-component mixture of a solvent and a solute or a dispersion of a solvent and colloids. Furthermore, we consider the solid object made up of the membrane to be regularly placed in a two-dimensional space with the help of periodic boundary conditions. The problem of the flow through a membrane is then reduced to the problem of the fluid flow around a single solid object. We are interested in a relative motion between the object and the fluid, which happens in either of the two situations: the object is static while the fluid is mobile, and the object is mobile while the fluid is static (Fig. 1). In colloidal solutions, the relative motion between the object's interface and the colloidal solution (dispersion) can represent two types of flows usually studied independently depending on which component is fixed in space. One distinguishes a) the solute-capillary flow induced by colloid concentration gradient (like osmosis and Marangoni flows) or b) the diffusio-phoresis of the object in a stagnant dispersion with a concentration gradient. A concentration gradient along the interface of an object results in a relative motion of the mixture with respect to the interface.

Diffusio-phoresis refers to the motion of particles in a solution, due to the concentration gradient of the solute. If the particles are large enough, then the concentration gradient results in significant changes of the solute concentration even across the size of the particle. In other words, the gradient exists along the particle-solution interface and drives the particle motion. A typical result of diffusio-phoresis is the motion of particles in the direction of the concentration gradient. If we change the reference frame and keep the object fixed, allowing the solution to flow around it, the phenomenon is typically called diffusioosmosis. In this case, the concentration gradient of the solute induces the flow along the object's surface, and we will refer to it, in a generic way, as interfacially driven flow (or solutocapillary flow). Correspondingly, in diffusio-osmosis, the solute will move against the direction of its concentration gradient. Maragoni flow is the fluid motion along the interface between two phases due to the gradient of the surface tension, which is caused by the concentration gradient in a mixture. The fluid moves in the direction of the concentration gradient, which is opposite to the osmotic flow.

In order to observe such interfacial flows, the system has to consist of at least three items: a two-component mixture and a)

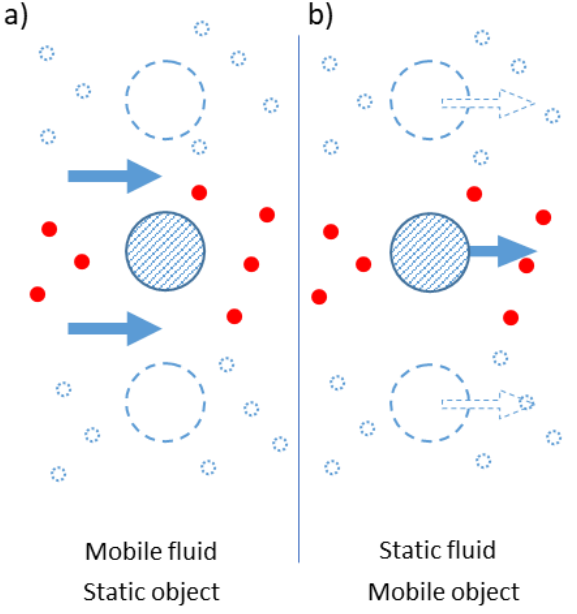

Fig. 1: 2D sketch of a multiphase flow with the interface between an object (large circles) and a dispersion (mixture) composed of a solvent (empty space in the figure) and colloids (solute) (small circles).

an object with an interface. The mixture may be a homogeneous solution or a colloidal dispersion of Brownian particles in the solvent, with a spatially varying solute concentration. The transport is driven by the gradient of the concentration along the interface of the object (a non-Brownian particle). In the following analysis, the objects are assimilated to the wall, which has a particular cylindrical shape in our work (Fig. 1). We will consider the objects to be fixed in space and we will investigate the flow patterns arising due to the concentration gradient of the solute (colloids) within the flow around the solid object.

This system represents a macroscopic configuration of both diffusio-phoresis and Marangoni flow phenomena. Since Marangoni flow and diffusio-osmosis result in an opposite transport behaviour, it is interesting to understand, which aspects of interfacial interactions are responsible for this. In particular, in the case of diffusio-osmosis the object plays the role of the internal walls of a membrane, while the mixture plays the role of an aqueous solution or colloidal dispersion.

Similarly, in the case of the Marangoni flow, the object provides a natural interface along which the gradient of the concentration is created. This model presents a unifying framework to study both of the aforementioned phenomena.

The crucial aspect of modelling of this system is the interactions between the mixture and the object. In this paper, the colloids interact with the interface and thus reside in the vicinity of the interface. In the case of attraction, the colloids are trapped, but not immobilized, by an attractive well: they are free to flow along the interface as it happens at a fluid/fluid interface. Molecular interactions between the components mimic the chemical properties of a real interface, thus allowing us to investigate its role in the interfacial driven transport. As we will see further, the type of these interactions determine the flow regime. In particular, if the interactions between the object and the mixture are purely repulsive, the flow is in the diffusioosmotic regime. In contrast, if the interactions have an attractive part, the flow is in the Marangoni regime. Thus, 
controlling the degree of the object interactions with the mixture, we can describe both phenomena.

The paper is organized as follows. Section 2 introduces the thermodynamic approach to describe the mass transfer and the flow of the mixture around the object. The next section presents the results of the simulation for diffusio-osmotic and Marangoni flows. In the last section related to discussion we generalize these results to unify interfacially driven transport phenomena: diffusio-phoresis, Marangoni flows and diffusio-osmosis flows.

\section{Thermodynamics of interfacially driven transport around an object}

Consider a mixture of two components (solution or dispersion) with a constant density. The mass fraction $\phi$ of the first component (solute or colloid) varies in space. Furthermore, let $\boldsymbol{\Pi}_{m}$ be the thermodynamic pressure, while $\boldsymbol{\tau}$ is the viscous pressure tensor. The fluid flow is characterized by the Péclet number $\boldsymbol{P e}$, which is a dimensionless form of the mixture velocity $\boldsymbol{u}_{m}=\phi \boldsymbol{u}_{c}+(1-\phi) \boldsymbol{u}_{f}$, which is the volume average of the solute (or colloids) velocity, $\boldsymbol{u}_{c}$ and of the solvent velocity, $\boldsymbol{u}_{f}$. The Péclet number is then also equal to the sum of the solute diffusion flux $\boldsymbol{J}_{\phi}$ and the solvent flux. The bold font of a variable indicates that it is a vectorial or tensorial quantity.

\subsection{Balance equations}

The fluid flow is described by the steady state balance equations. According to the previous development ${ }^{19}$, the nondimensionalised set of equation to solve in stationary state is:

$$
\begin{gathered}
\nabla \cdot \boldsymbol{P} \boldsymbol{e}=0 \\
\nabla \cdot \boldsymbol{J}_{\boldsymbol{\phi}}=0 \\
\nabla \cdot\left(\boldsymbol{\Pi}_{m}+\boldsymbol{\tau}\right)=0
\end{gathered}
$$

The first two equations are the mass conservation equations for the mixture and the solute (or colloid) respectively. The third equation is the incompressible Navier Stokes equation, with a hydrodynamic force term due to the overall pressure gradient, $\nabla \cdot \boldsymbol{\Pi}_{m}$, that applies on the mixture, and the viscous dissipation term $\nabla \cdot \boldsymbol{\tau}$. Furthermore, the material time derivative and consequently the change of momentum due to convection proportional to $\mathbf{v} \cdot \nabla \mathbf{v}$ are neglected, as we consider a flow regime with low Reynolds number.

\subsection{Equation of state}

From a thermodynamic perspective, we can distinguish different contributions to the thermodynamic pressure associated with molecular interactions between different components. In particular, for the three components (solvent $f$, solute (or colloid) $c$, object (or interface) $i$ ) we have five types of interactions: $f f, f c$, if, ic, $c c$ (ii interactions are not considered, as the solid objects are placed far away from each other and immobile).

In this paper, we follow the physical description of the Suspension Balance Model 19-21. In that model the first component in the mixture is a colloid while the second component is referred to as a fluid. In such a two-fluid model the thermodynamic equation of state differs from the one for a homogeneous solution. In particular, the overall mixture pressure gradient, $\nabla \cdot \boldsymbol{\Pi}_{m}$, consists of three contributions: i) the gradient of fluid pressure (due to the fluid-fluid interactions), $\nabla p_{f f}$, ii) the local force induced by colloid-object interactions, $\nabla \cdot \boldsymbol{\Pi}_{i c}$ that applies on the colloid volume $\phi$, iii) the fluid-object interactions, $\nabla \cdot \boldsymbol{\Pi}_{\text {if }}$ that holds for the fluid volume, $1-\phi$ :

$$
\nabla \cdot \boldsymbol{\Pi}_{m}=\nabla p_{f f}+\phi \nabla \cdot \boldsymbol{\Pi}_{i c}+(1-\phi) \nabla \cdot \boldsymbol{\Pi}_{i f}
$$

The first term relates to an "internal" force due to variation of the fluid pressure while the two last ones relate to an 'external' force from the interface (the interface is an external object to the mixture) which acts on both colloid, $\phi \nabla \cdot \boldsymbol{\Pi}_{i c}$, and the fluid $(1-\phi) \nabla \cdot \boldsymbol{\Pi}_{i f}$. This thermodynamic equation for the pressure gradient specifies the nature of the molecular interaction in the system. The pressure tensor $\boldsymbol{\Pi}_{m}$ is anisotropic (it is no longer a scalar quantity) close to the interface.

It has to be noted that the osmotic pressure $\boldsymbol{\Pi}_{c c}$ (that describes colloid-colloid interactions) does not contribute to the gradient of the thermodynamic pressure $\boldsymbol{\Pi}_{m}$. The reason for that is that the osmotic pressure does not affect the mixture's momentum directly. As discussed in earlier works ${ }^{20,22}$, the osmotic pressure gradient induces a dissipative force on the colloidal particles, which puts a reaction force on the fluid. For the mixture, these two contributions cancel each other. The contribution of the osmotic pressure will be evident later in Equation (8) which describes the colloid diffusion flux $\boldsymbol{J}_{\phi}$. In this paper, for the sake of simplicity and in order to unravel the mechanisms, the osmotic pressure will be calculated from the van't Hoff law for an ideal dispersion: the colloid-colloid interactions being neglected, the diffusion coefficient remains independent of the colloid volume fraction. The ideal dispersion hypothesis is considered since we are interested in the effect of the colloidinterface interactions on the fluid dynamics. The model then represents the flow of a rather dilute dispersion close to an interacting interface. Similarly, the colloid-fluid interactions $\boldsymbol{\Pi}_{c f}$ contribute to the irreversible behaviour of the mixture, which is discussed in the following section.

The fluid dynamics approach to the problem considered would be to solve Equations (1) to (3) in specific geometries by introducing solid walls as boundary conditions with no-slipping conditions. In this paper, another approach is chosen: the equations are solved for the entire spatial domain, but with a local penalization method to account for the presence of solid walls. In Equation (4), a term $\boldsymbol{\Pi}_{\text {if }}$ is thus added to penalize the flow in the solid domain described by Equation (3). The term $\boldsymbol{\Pi}_{\text {if }}$ physically expresses the fluid-object interaction that forces the flow away from the interface. This way of writing the Equation (4) has the advantage of treating the wall interactions similarly: the presence of the narrow channel in the flow is represented through the interactions that the object interface exerts on the solvent/fluid, $\boldsymbol{\Pi}_{i f}$, together with the interaction it exerts on the solute/colloid, $\boldsymbol{\Pi}_{i c}{ }^{23-24}$. These interactions are a function of the distance to the object, which is determined through a level set method. The penalization for the solvent is a very stiff 
exponential function that applies in a very thin interfacial layer close to the interface (Fig. 2). To be negligible, the interfacial



Fig. 2: a) 2D representation of the cylinder geometry in the case of colloid-interface repulsion $\left(a_{t t}=0\right)$ : the solid is represented in brown and the colloid-wall interaction, $\Pi_{i c}$, magnitude with the colour map. b) Colloid - interface interaction profile as a function of the distance to the cylinder, $d$, for pure repulsion $\left(a_{t t}=0\right)$ and several attractionrepulsion cases $\left(a_{t t}>0\right)$.

layer for solvent-interface interactions is less than one tenth of the interfacial layer for solute-interface interactions. The interaction between the solute and the object are also represented by an exponentially decreasing function similar to the one that could be obtained by the DLVO theory ${ }^{19}$. The scalar component of $\boldsymbol{\Pi}_{i c}$ is:

$$
\Pi_{i c}=\left(1+a_{t t}\right) k e^{-\frac{d}{l}}-a_{t t} k e^{-\frac{d}{2 l}}
$$

where $a_{t t}$ is the attractive parameter. It has been defined to study the role of long-range attraction by combining a negative (attractive) and positive (repulsive) interaction. The decay length for the attraction is fixed at $2 /$ to describe a minimum in interaction at long range. This term, the last one in Equation (5), corresponds to DLVO interaction profile with a secondary minimum. The short-range repulsion is necessary to keep physical consistency with volume exclusion of the colloids by the object.

\subsection{Irreversible processes}

The thermodynamic contributions to the pressure considered in the previous subsection exist even in equilibrium, i.e. when the mixture is at rest. In contrast, irreversible contributions are present due to the mixture motion. Among these irreversible contributions we distinguish the diffusive flux $\boldsymbol{J}_{\phi}$., which is responsible for the relative motion of the colloids with respect to the fluid, as well as the viscous pressure tensor which is responsible for spatial variation of the mixture's velocity.

The viscous pressure tensor and the diffusive flux, which describe irreversible processes, are obtained from the following linear force-flux relations of non-equilibrium thermodynamics:

$$
\begin{gathered}
\boldsymbol{\tau}=-\mu(\phi)\left(\nabla \boldsymbol{P} \boldsymbol{e}+\nabla \boldsymbol{P} \boldsymbol{e}^{T}\right) \\
\boldsymbol{J}_{\boldsymbol{\phi}}=-D(\phi) \nabla \psi_{c}
\end{gathered}
$$

where $\psi_{c}$ is the chemical potentials of the colloids relative to the fluid. Here $\nabla \boldsymbol{P e}$ and $\nabla \psi_{c}$ are the driving forces of viscous transport and mixing respectively. Furthermore, $\mu(\phi)>0$ and $D(\phi)>0$ are the viscosity coefficient and the diffusion coefficient, which are the transport coefficients of the corresponding irreversible processes. Equations (6) and (7) have the simplest form of decoupled Newton's law and Fick's law when the temperature is constant. Furthermore, for constant viscosity and an incompressible fluid, the $\nabla \boldsymbol{P} \boldsymbol{e}^{T}$ term becomes zero after taking the divergence in Equation (3).

According to the Suspension Balance Model 19-21,25 the irreversible process of mixing is governed by Stokes' law, so the colloidal particles which have the mobility $m(\phi)$, experience a negative drag force which is proportional to the velocity difference between the colloidal particle and the mixture, namely, $\boldsymbol{F}_{c}=-\left(\boldsymbol{u}_{c}-\boldsymbol{u}_{m}\right) / m(\phi)$. In the stationary state the drag forces acting on $n=\phi / V_{p}$ particles of volume $V_{p}$ per $\mathrm{m}^{3}$ are balanced by the gradient of the thermodynamic pressure, $n \boldsymbol{F}_{c}=\nabla \cdot \boldsymbol{\Pi}_{c c}+\phi \nabla \cdot \boldsymbol{\Pi}_{i c}$, so Equation (7) can be rewritten as

$$
\boldsymbol{J}_{\phi}=\phi \boldsymbol{P e}-K(\phi)\left(\nabla \cdot \boldsymbol{\Pi}_{c c}+\phi \nabla \cdot \boldsymbol{\Pi}_{i c}\right)
$$

where $K(\phi)=6 \pi \mu a m(\phi)>0$ is the settling hindrance coefficient, which is equal to 1 for dilute dispersion.

The set of balance equations (1) to (3) with a thermodynamic viewpoint described by equations of state (4) and (5) and irreversible process equations (6) to (8) is similar to the one obtained from a mechanical approach based on the suspension balance model ${ }^{19,20}$. However, instead of aggregating all terms, here one distinguishes the contributions from mechanistic balance equations and irreversible thermodynamics. This allows for more flexibility and better understanding of assumptions. For example, Newton's law equation (6) can be written with a different functional form for the shear rate, a dependency of the viscosity coefficient on position, a nonlinear expression or additional heat terms due to thermodynamic coupling with heat transfer. These effects could be investigated in future studies.

\subsection{Dimensionless variables}

In Table 1, the dimensionless variables are defined as in the previous work ${ }^{19}$ and their links with the dimensional variables are quantified. The non-dimensional velocity and the flux are obtained by dividing the corresponding dimensional terms by the diffusion force, so that, in particular, the advection term becomes a Péclet number in Equations (1) to (3). For the nondimensionalization, the diffusion coefficient is considered to be of a dilute condition, $D_{0}=m_{0} k_{B} T$, where $m_{0}$ is the mobility of a dilue solute having a volume, $V_{p}$. The corresponding Reynolds number is thus the Péclet number divided by the Schmidt number, Sc.

The spatial units are scaled with the characteristic length scale $\delta=2 \cdot 10^{-6} \mathrm{~m}$ (Fig.2). The set of data corresponds then to a dispersion of colloidal particles with the diameter $10 \mathrm{~nm}$ around a cylinder object with a $400 \mathrm{~nm}$ diameter. Such a size ratio ensures that the dispersion is treated as a continuous medium and the Eulerian approach is correctly used. Under these conditions, the non-dimensional viscosity is equal to $5.5510^{-6}$. The dependence of viscosity on the volume fraction is not taken into account.

For the interactions, the decay length $l$ is taken to be equal 0.048 and the energy parameter $k$ (which is equal to the maximum value of the energy at the wall), is fixed at 96.9. These values are defined to be close to those calculated for $10 \mathrm{~nm}$ spheres dispersed in a $10^{-5} \mathrm{M}$ solution with a zeta potential of $80 \mathrm{mV}$ for both particles and walls. The solute -interface energy 
map and interaction profiles obtained with Equation (5) are plotted in Fig. 2. The attractive-repulsive cases $\left(a_{t t}>0\right)$ display a distinct minimum of $\boldsymbol{\Pi}_{i c}$. For the $\left(a_{t t}=00\right)$ case, the minimum is barely distinguishable and we will observe later a behaviour alike the pure repulsive interaction case. Equation (5) is also used to define the solvent/fluid interaction, $\boldsymbol{\Pi}_{i f}$, with a decay length taken at 0.01 to be small compared to the decay length for the colloid/interface interaction.

Other geometrical configurations (i.e. different ratios of the decay length, the cylinder size and the interstitial distance between two cylinders) represent membranes with different internal structures (e.g. with smaller pore size). The effect of the interfacially driven flow depends on these ratios, while the observed mechanisms remain the same.

\section{Results}

In this paper, the calculation is done for colloid-interface interaction defined for a pure repulsive case $\left(a_{t t}=0\right)$ and for several attractive-repulsive cases $\left(a_{t t}>0\right)$. The set of equations is solved with the partial differential equation solver Fipy ${ }^{26}$ (finite element volume) implemented on the Python platform Canopy (Enthought, Austin). Simulations are performed with periodic conditions on the top and bottom boundaries: this corresponds to the flow through an array of cylinders as depicted in Fig. 1. The non-dimensional horizontal size of the box is 2, which corresponds to $2.10^{-6} \mathrm{~m}$. Simulations presented in the next sections are realised in a solute concentration gradient. The concentration gradient is constant through the system with the volume fraction equal to 0.01 on the left boundary and to 0 on the right boundary. The hydrodynamic and the mass transfer are calculated for different Péclet numbers and in the case of i) pure repulsion between the solute and the object (section 3.1) and ii) attraction-repulsion profile (section 3.2 to 3.4 ).

\subsection{Simulation of diffusio-osmosis and diffusio-phoresis for object} repealing colloids

The goal of the simulation is to describe the mobile flow of diffusio-osmosis ${ }^{29}$ or diffusio-phoresis $27,28,30$ around a circular static object in presence of a gradient in colloids concentration. The solute - interface interaction is repulsive $\left(a_{t t}=0\right)$ (Fig. 2).

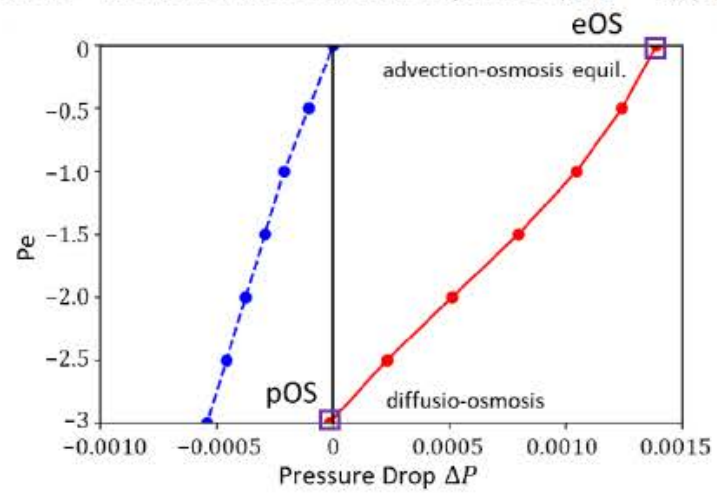

Fig. 3: Péclet number as a function of the pressure drop, $\Delta P$, across the channel (full red line from advection-osmosis equilibrium [eOS] to pure diffusio-osmosis [pOS]). The dotted line represents the same data when only the solvent is filtered or in absence of colloid concentration gradient.

Within the fixed concentration gradient the simulations describe the mass flux (the diffusion from high to low concentration) and the flow induced by the interactions of the colloid at the interface, i.e. the diffusio-osmosis phenomena. The net inlet flow (here a Péclet number) is fixed and the simulation describes the local flow around the sphere and allows the calculation of the pressure gradient needed to ensure the net inlet flow. The simulations are performed in transient mode, but here only the steady state simulations are discussed

The calculations allow determining the relationship between the pressure drop due to the flow $\Delta P$ and the flow at steady state. Fig. 3 plots the Péclet number as a function of the pressure drop. The red full curve on the right refers to simulations that are performed for negative Péclet numbers $\boldsymbol{P} \boldsymbol{e}<0$, corresponding to a flow opposite to the concentration gradient so as to stay in osmosis conditions. Conversely, reverse osmosis conditions are obtained for positive Péclet numbers and discussed in section 3.4. The pressure drop for pure water (dotted line) is proportional to the velocity as expected ${ }^{31}$.

As shown in Fig. 3, the pressure drop varies between two extremal values of osmosis, from the flow at equilibrium (eOS)

Table 1: The dimensionless quantities used to define the dynamic osmotic or Marangoni flow problem. The correspondence with the dimensional quantities is given for a colloid radius, a $=10^{-8} \mathrm{~m}$, a characteristic length scale for the geometry (Figure 2), $\delta=2.10^{-6} \mathrm{~m}$, a mixture viscosity, $\mu=10^{-3} \mathrm{~Pa} . \mathrm{s}$, and a temperature T=298 K

\begin{tabular}{|c|c|c|c|}
\hline Physical quantity & Dimensionless quantity & Physical relationship & Numerical relationship \\
\hline Velocity, $\boldsymbol{u}_{\boldsymbol{m}}$ & $\begin{array}{c}\text { Péclet number, } P \boldsymbol{P} \\
\text { Reynolds number, } R e\end{array}$ & $\begin{aligned} \boldsymbol{P e} & =\frac{\delta}{m_{0} k T} \boldsymbol{u}_{m} \\
R e & =|\boldsymbol{P e}| / \mathrm{Sc}\end{aligned}$ & $\begin{array}{c}\boldsymbol{u}_{m}(m / s)=1.09 \cdot 10^{-5} \boldsymbol{P e} \\
\boldsymbol{u}_{m}(m / s)=0.5 R e\end{array}$ \\
\hline Mass flux, $\tilde{J}_{\phi}$ & Mass flux, $\boldsymbol{J}_{\phi}$ & $\boldsymbol{J}_{\boldsymbol{\phi}}=\frac{\delta}{m_{0} k T} \boldsymbol{u}_{c} \boldsymbol{\phi}$ & $\boldsymbol{u}_{c}(\mathrm{~m} / \mathrm{s})=1.0910^{-5} \boldsymbol{J}_{\phi} / \phi$ \\
\hline Pressures, $\widetilde{\boldsymbol{p}}, \tilde{\boldsymbol{\tau}}, \widetilde{\boldsymbol{\Pi}}$ & Pressures, $\boldsymbol{p}, \tau, \Pi$ & $\boldsymbol{\Pi}=\frac{V_{p}}{k T} \widetilde{\boldsymbol{\Pi}}$ & $\widetilde{\boldsymbol{\Pi}}(P a)=982 \boldsymbol{\Pi}$ \\
\hline Viscosity, $\tilde{\boldsymbol{\mu}}$ & Viscosity, $\mu$ & $\mu=\frac{2 a^{2}}{9 \delta^{2}} \frac{\tilde{\mu}}{\mu_{0}}$ & $\tilde{\mu}(P a \cdot s)=1.810^{5} \tilde{\mu}_{0} \mu$ \\
\hline Mobility, $\boldsymbol{m}$ & Settling hindrance coefficient, $K$ & $K(\phi)=\frac{m(\phi)}{m_{0}}$ & $m\left(k g^{-1} \cdot s\right)=5.3110^{9} K(\phi)$ \\
\hline
\end{tabular}


(when the net flow is zero, $\boldsymbol{P e}=0$ ) to the pure osmosis conditions (pOS) (where the pressure drop is zero, i.e. no external mechanical forces applied). The latter condition corresponds not only to the diffusio-osmosis case (flow of the liquid around a non-mobile object due to the concentration difference) but also to the diffusio-phoresis case (motion of the object in a stagnant fluid due to the concentration gradient). The velocity around $\boldsymbol{P} \boldsymbol{e}=-30$ in Fig. 3 can be considered as the maximum relative velocity between the object and the fluid: this is the fluid velocity if the object is immobile or, conversely, the object velocity when the fluid is at rest.

Under equilibrium conditions $(\boldsymbol{P} \boldsymbol{e}=0)$, the pressure drop is also equivalent to the drag force that one should apply on the object to keep it immobile. In the present case of colloid-object repulsion considered, the pressure drop is positive and around 0.0013 in Fig. 3. The force due to osmosis is then positive and will induce a displacement of the object towards the low solute concentration (diffusio-phoresis) ${ }^{10-32}$. This value of the pressure drop in the equilibrium condition is equal to the product, $\sigma \Delta \Pi$, where $\sigma$ is the Staverman reflection coefficient. The simulation allows us then to determine the value of $\sigma$ at 0.13 (recall that $\Delta \Pi=0.01$ ) As previously discussed with an 1D approach ${ }^{22}$, the reflection coefficient can be defined as, $\sigma=1-\Phi$, where $\Phi$ is the partition coefficient that is related to the repulsive potential barrier between the object.

These global flows are the consequences of local flow that are also provided by the simulations. Fig. 4 plots the $x$ and $y$ component of the local flow velocity and the stream lines around the objects for the two extreme conditions: for pure osmosis and advection-osmosis equilibrium.

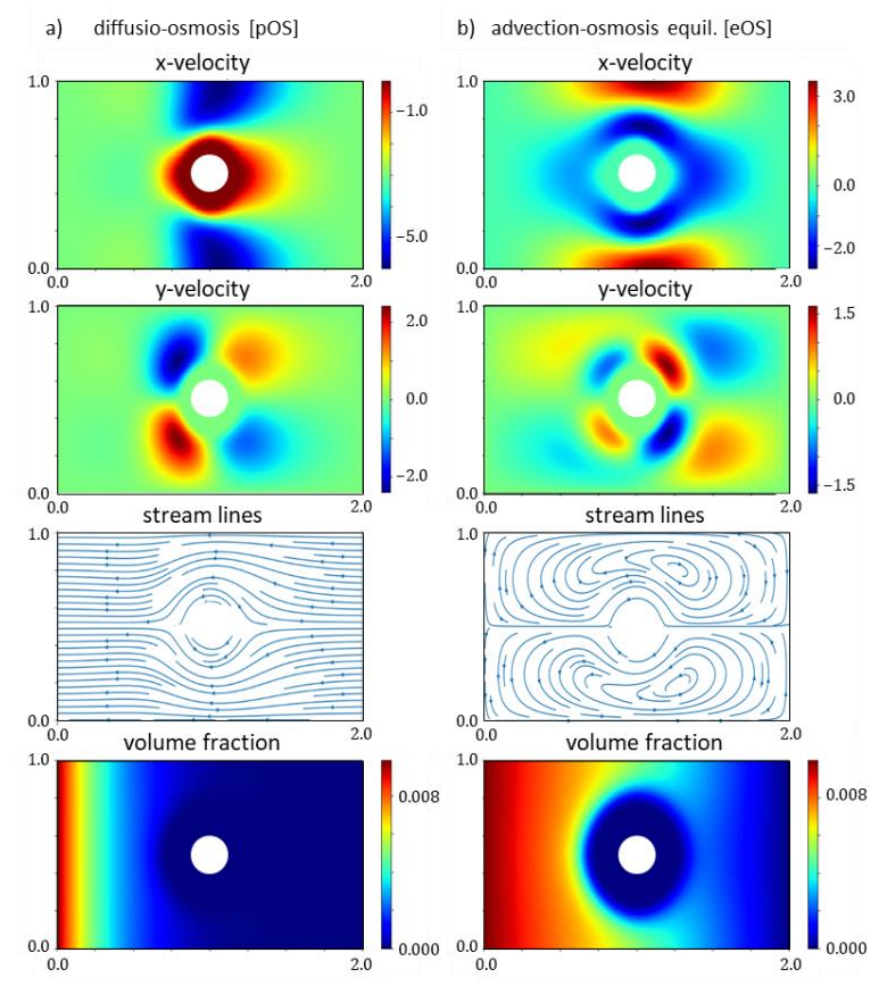

Fig. 4: Local flow at steady state a) for the pure diffusio-osmosis case [pOS], $\Delta P=0$ and b) for the advection-osmosis equilibrium [eOS], $\boldsymbol{P} \boldsymbol{e}=0$. The magnitude of $\mathrm{x}$ and $\mathrm{y}$ velocities (represented by dimensionless local Péclet number) are displayed in the first and second diagrams, the corresponding stream lines in the third one and the volume fraction in the fourth diagram.

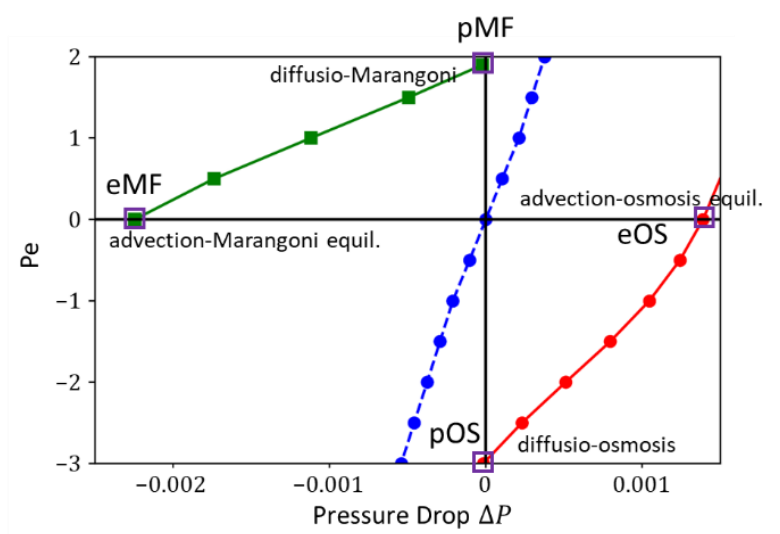

Figure 5: Péclet number as a function of the pressure drop, $\Delta P$, across the channel (full green line from advection Marangoni equilibrium eMF to pure diffusio-Marangoni flow pMF). The blue dotted line represents the same data when only the solvent is filtered or in absence of colloid concentration gradient. The full red line is taken from Fig. 3.

During osmosis (Fig. 4a), the stream lines are nearly parallel, being deviated around the object and the flow is similar to a creeping flow. In the interstitial zone between cylinders, the velocity is rather constant and like a plug flow. When the osmotic flow is counter-balanced by the advection $(\boldsymbol{P e}=0)$ (Fig. 4b), the flow exhibits a secondary flow ${ }^{19}$, with a flow toward the left close to the object interface (because of diffusioosmosis directed toward high solute concentration) and a return flow to the right in the interstitial zone between cylinders. The net flow across the section is zero.

It has to be noted that during pure osmosis (pOS) no net force is exerted on the object: the negative drag force exerted by the flow is compensated by the positive interfacial force (given by $\left.\phi \nabla \cdot \boldsymbol{\Pi}_{i c}\right)$ due to the higher concentration on the left side. Unlike in the case of a forced convection flow, the object is then not compressed by the flow. This can have important consequences in processes and can explain the difference in behavior in process like forward osmosis (where the flow does not exert a compression on the membrane or on the accumulated cake) and reverse osmosis (where the flow compresses the membrane and the accumulated cake) ${ }^{33}$.

\subsection{Simulation of diffusio-marangoni and diffusio-phoresis for object attracting colloids}

Similar simulations can also be run with solute-interface attraction within a range near the interface, completed with a repulsion closer to the interface wall (Fig. $2 b, a_{t t}>0$ ). The interaction profile now exhibits a negative attractive minimum. Fig. 5 presents the flow (Péclet number) as a function of the pressure drop for the solute - object interface attractive conditions $\left(a_{t t}=0.02\right)$. It should be noted that in this case the results (full green curve) are displayed in the quadrant opposite to the osmosis results reproduced from Fig. 3 (full red curve) because of the flow inversion. Indeed, Fig. 5 exemplifies that our model is able to represent both osmosis and Marangoni phenomena. 
In this case, the simulations predict that the presence of the attractive part of the colloid-interface potential changes the direction of the flow in the gradient of solute concentration. Indeed, contrary to the diffusio-osmosis discussed in the previous subsection and occurring in presence of soluteinterface repulsion, the fluid is now driven toward the low solute concentration when $\boldsymbol{P e}>0$. This is due to the attractive term. It can be considered as a Marangoni flow and we will refer to it as the diffusio-Marangoni flow (which is sometimes also called solute-capillary or soluto-Marangoni flow in the literature). Indeed, the diffusio-Marangoni flow is directed toward the zone of low solute concentration that also represents the zone of higher surface tension for a solute, which has an affinity for the interface like a surfactant.

The observed direction of the local flow is coherent with the classical thermodynamic approach. The nature of the colloidinterface interactions changes the water pressure ${ }^{24}$, the water activity 22 and the surface tension according to the solution theory ${ }^{34}$. The interaction of colloids with the interface leads to an anisotropy in pressure close to the interface. This stress anisotropy normal to a free fluid surface leads to an interfacial (capillary) stress that leads to a surface tension, $\gamma$. When the concentration of colloids interacting with repulsion (resp. attraction) with the interface increases, the water pressure and the water activity close to the interface decreases (resp. increases) and the surface tension increases (resp. decreases)

22. Simulations presented in the paper satisfy the second law of thermodynamics: the flow of water is always towards the low water activity or towards the high surface tension i.e. in the direction of high concentration of the surfactant (the generic

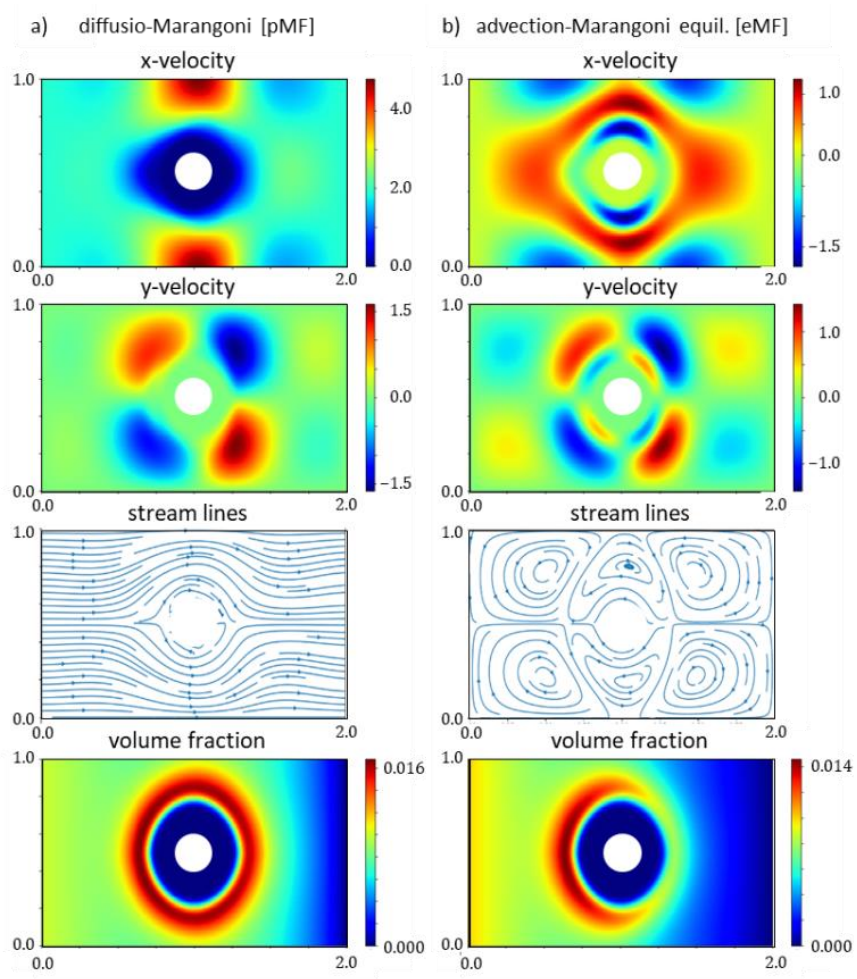

Fig. 6: Local flow at steady state a) diffusio-Marangoni case [pMF], with $\Delta P=0$ and $\boldsymbol{P} \boldsymbol{e}=$ 1.9. b) advection-marangoni equilibrium [eMF], with $\boldsymbol{P} \boldsymbol{e}=0$ and $\Delta P=-0.00225$. The magnitude of $x$ and $y$ velocities (represented by dimensionless local Péclet number) are displayed in the first and second diagrams, the corresponding stream lines in the third one and the volume fraction in the fourth diagram.

surfactant case corresponds to $d \gamma / d c<0$ ) and in the direction of low concentration for repelling colloids (where $d \gamma / d c>0$ ) ${ }^{35}$

Fig. 6 plots the $x$ and $y$ component of the local flow velocity and the stream lines around the spheres for the two extreme conditions encountered in the case of attractive - repulsive solute - interface interactions, namely diffusio-Marangoni (pMF) and advection-Marangoni equilibrium (eMF) conditions. The simulations have been performed for the same conditions as in the previous section, for the pressure drop ranging between the case of flow equilibrium $(\boldsymbol{P} \boldsymbol{e}=0)$ and zero (net force of the object equal to zero). For the case $\Delta P=0$, the pure Marangoni Flow velocity is equal to $\boldsymbol{P e}=1.9$. When $\boldsymbol{P e}=0$, we observe the advection-Marangoni equilibrium.

Secondary flows corresponding to these two extreme cases are displayed in Fig. 6 stream lines diagrams. Overall, the flow is more complex than the pure repulsive case (section 3.1), mainly because the interaction profile presents here both a long range attraction and a short range repulsion.

In the diffusio-Marangoni regime (Fig. 6a), the flow is in the opposite direction to the one observed when there is a repulsive solute - interface interaction only (Fig. 4a). The flow is then directed toward the zone of low solute concentration. The $x$ velocity is almost always positive in the whole region. This flow is a solute-capillary Marangoni flow with a flow toward the zone where solute having an affinity with the interface are less concentrated (or zone of higher surface tension).

The pattern for the $x$ and $y$ velocities looks like to the one obtained for a regular plug flow around a cylinder (as sketched for pure osmosis flow in Fig. 4a), but is inverted in sign. The main deviation to the plug flow is due to the repulsion at short distance that can superimpose local osmotic flow.

At the Advection - Marangoni flow equilibrium condition (Fig. $6 b)$, there are three layers of fluid with alternate velocity in the interstitial zone between the objects from neighbouring unit cells. Close to the object wall, there exists a zone with a low negative velocity corresponding to a diffusio-osmotic flow, because of the short range repulsion. Then, because of the long range attraction, there is a zone of large positive fluid velocity (toward the low solute concentration) corresponding to the diffusio-Marangoni flow. Finally, there is a third layer with a negative back flow that ensures the zero flow condition at the equilibrium flow conditions that hold here. These fluid layers with alternate flow direction lead to the development of secondary flows.

When analysing the pressure and the volume fraction field in these cases, we can note that the overall flows are mainly due to the distortion of the mass accumulation ring in the short range attraction zone around the circular object.

\subsection{Transition between osmosis and Marangoni flows: neutralized flow}

Varying the magnitude of the attractive term, we can investigate the transition between Marangoni and osmotic flows. Fig. 7 plots the variation of the Péclet number as a 
function of the pressure drop for intermediate value of the attraction term in the interaction potential. The parameter $a_{t t}$



Fig. 7: Variation of the Péclet number as a function of the pressure drop, $\Delta P$, for different values of the attractive parameter, from $a_{t t}=0$ (pure repulsion) to $a_{t t}=0.2$ (significant attraction). Labelled points represent some specific flow configuration, detailed in Fig. 4, 6, 8 and 9.

is varied in Equation (5) from 0 (pure repulsion) to 0.2 (for significant attraction) and the corresponding interaction profiles and the values of the attractive well are shown in Fig. 2b.

Nearly neutral conditions (NE) can be obtained for an intermediate value of $a_{t t}=0.1$. In these conditions, the Marangoni flow due to attraction compensates the osmotic flow due to repulsion and the flow is then close to the one obtained for pure water (blue line in absence of colloid) or for the flow of colloid that do not interact with the object. The flow map for the neutral case $\left(a_{t t}=0.1\right)$ is shown in Figure 8 .

3.4 Reverse-Osmosis, Reverse-Marangoni and Inverted-Marangoni flows: generalizing examples

In Fig. 7, when the Péclet is positive (resp. negative), the gradients of pressure and of concentration are in the same direction (resp. opposite): the flow is advecting high (resp. zero) colloid concentration toward the object. When the pressure drop $\Delta P$ is larger (resp. smaller) to the one for pure water $\Delta P_{w}$, (blue line), the interfacially driven transport increases (resp. reduces) the drag force. Hence, four quadrants can be considered according to Péclet and pressure drop values:

- In the top right quadrant $\boldsymbol{P e}>0$ and $\Delta P>\Delta P_{w}$, the interfacially driven transport is contrary to the main flow that is in the direction of low concentration: this corresponds mainly to reverse osmosis (rOS).

- In the bottom right quadrant $\boldsymbol{P} \boldsymbol{e}<0$ and $\Delta P>\Delta P_{w}$, the interfacially driven transport goes along the main flow that is in the direction of high concentration: it corresponds to an osmotic flow (OS)toward high concentration.

- In the top left quadrant $\boldsymbol{P e}>0$ and $\Delta P<\Delta P_{w}$, the interfacially driven transport goes along the main flow that is in the direction of low concentration: it corresponds to a Marangoni flow (MF) toward low concentration.

- In the bottom left quadrant $\boldsymbol{P} \boldsymbol{e}<0$ and $\Delta P>\Delta P_{w}$, the interfacially driven transport is opposite to the main flow that is in the direction of low concentration: it corresponds to a reverse-Marangoni flow (rMF) to use the same terminology than for reverse-osmosis.

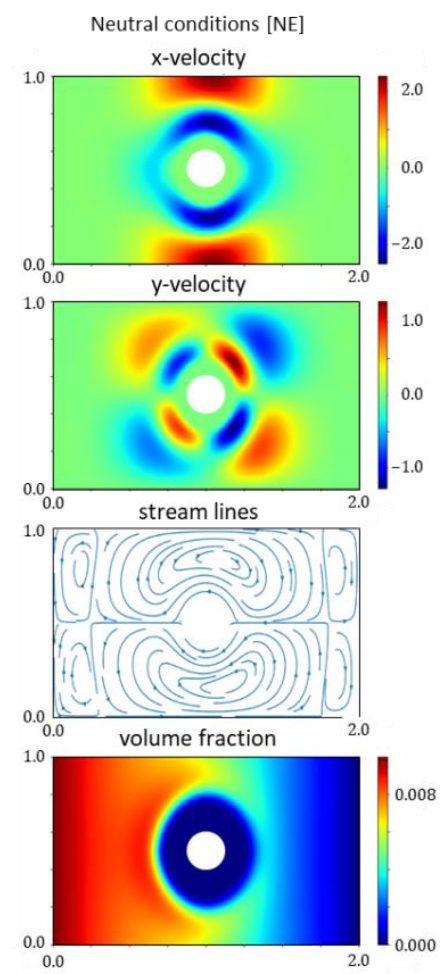

Fig. 8: Local flow at steady state for the "neutralized Marangoni-osmotic" flow (NE) $\left(a_{t t}=0.1\right)$ at $\boldsymbol{P} \boldsymbol{e}=0$. The magnitude of $\mathrm{x}$ and $\mathrm{y}$ velocities (represented by dimensionless local Péclet number) are displayed in the first and second diagrams, the corresponding stream lines in the third one and the volume fraction in the fourth diagram.

The flow maps are shown in Figure 9. When repulsion is predominant (i.e. for $a_{t t} \leq 0.05$ ), the interfacially driven transport is directed toward high colloid concentration and then can be related to the osmosis mechanism. For $\boldsymbol{P e}>0$ (corresponding to reverse osmosis situation) the osmosis leads to an increase of the pressure drop compared to the one obtained for pure water. The additional pressure drop due to the osmosis, also called counter-osmotic pressure, increases when the magnitude of repulsion increases or when the Péclet number is higher. This is due to a more important accumulation in the upstream zone. When the flow is opposite to the concentration gradient $(\boldsymbol{P e}<0)$, the osmosis is in the direction of the flow and then contribute to a reduction of the pressure drop.

When attraction between the object and the colloids become important, Marangoni flow (from high to low colloid concentration) predominates. When the Péclet is small and positive, the highest concentration is in the upstream zone and the Marangoni flow is in the same direction than the main flow (insert MF in Fig. 7 for $\boldsymbol{P e}=1$ and $a_{t t}=0.2$ ). The Marangoni leads then to a reduction of the pressure drop. For larger Péclet number, it has to be noted that Marangoni flow can lead to pressure drop higher than the one obtained for pure water. This is because the direction of the Marangoni flow is changed by the flow: the sweeping out of the concentrated attracted zone 

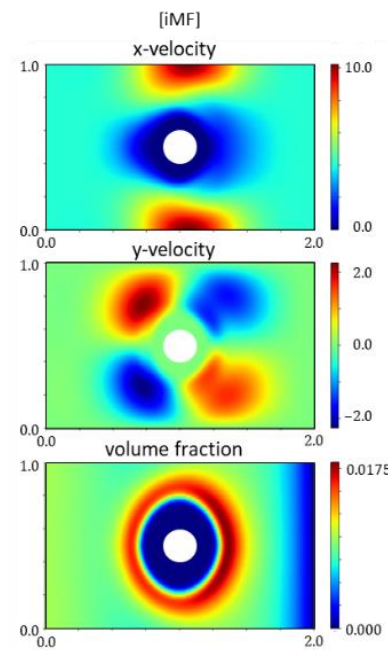

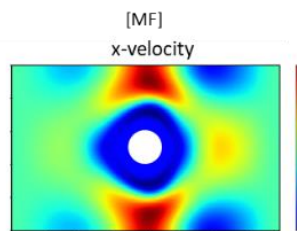

$y$-velocity

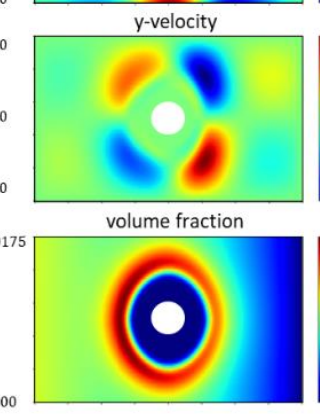

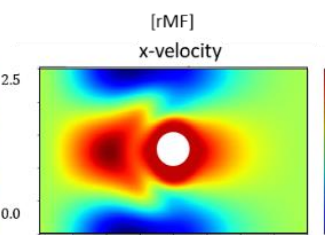

y-velocity

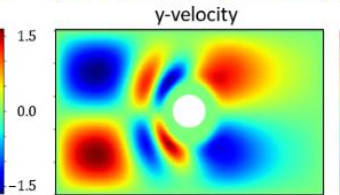

volume fraction

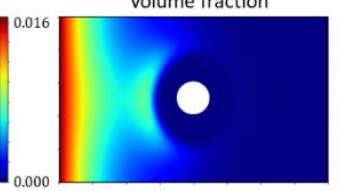



y-velocity

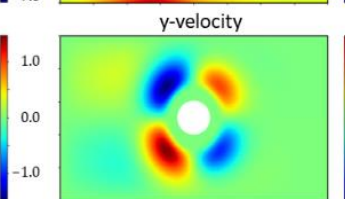

volume fraction

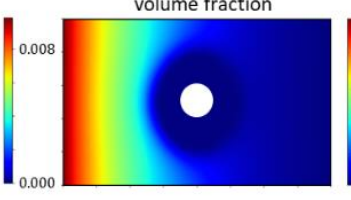

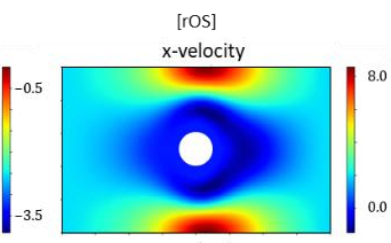

y-velocity

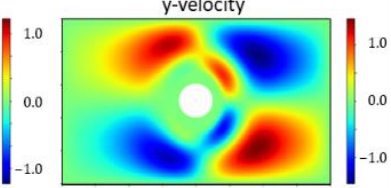

volume fraction

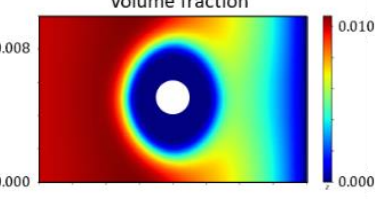

Fig. 9: 2D volume fraction (bottom line) and velocity maps ( $\mathrm{x}$ velocity top line and y velocity middle line) for significant cases in the different quadrant (from left to right Inverted Marangoni Flow (iMF), Marangoni-flow (MF), Reverse Marangoni Flow (rMF), Osmosis (OS) and reverse osmosis (rOS) corresponding to flow conditions reported in Fig. 7).

of colloids around the object by the flow. For such conditions (insert iMF in Fig. 7 for $\boldsymbol{P e}=4$ and $a_{t t}=0.2$ ), the concentration in colloids is highest in the downstream zone and then leads to a Marangoni opposite to the flow. This is typically the case that is encountered when a bubble (than can be assimilated to the object we use in simulation) is rising up in a solution with surfactant (the surfactant can be associated to the colloids being attracted by the object). Because of the flow, surfactants are accumulated in the downstream zone of the bubble and lead to a Marangoni flow opposite to the rising velocity that, in turn, increases the drag force on the bubble ${ }^{36}$.

For negative Péclet number (insert rMF in Fig. 7 for $\boldsymbol{P e}=-3$ and $a_{t t}=0.2$ ) i.e. when the flow is in the direction of the highest colloid concentration, the Marangoni flow is opposite to the main flow (this situation is called reverse-Marangoni in the Fig. 7 to use the same terminology than for reverse-osmosis). It has to be noted in Fig. 7 that for high negative flow $P \boldsymbol{e} \ll 0$, the flow behavior becomes close to the one obtained for a single-component fluid, i.e. without colloidal particles (water blue line in Fig. 7). This is mainly because the flow is sweeping out the colloid concentration gradient and brings a dispersion without colloids toward the object. The absence of significant colloid-interface interactions leads to a flow behaviour close to the one for pure fluid. Such a behaviour is not observed for large positive Péclet number. For these conditions, the flow is advecting high colloid concentration toward the object that lead either to specific osmotic or Marangoni flows, depending on the colloid - interface interactions.

\section{Unification of osmosis, Marangoni and diffusio- phoresis phenomena}

Despite the fact that both Marangoni flow and diffusio-osmosis are considered to be phenomena related to interfacially driven transport, no unifying approach, which explains the mechanism of this relationship for simple non-polar fluids, is immediately obvious. This paper fills this gap, suggesting a tractable framework in which both Marangoni flow and diffusio-osmosis



Fig. 10: Interfacially driven transport induced by the interaction of colloids with an interface. Four mechanisms result from the interfacially driven transport according to the nature of the interactions (mainly repulsive or attractive) and to the mobilities of the fluid or the interface. 
emerge naturally because of the variation of a single parameter This parameter controls the character of the interactions between the colloidal particles dispersed in a fluid and a solid object along which the mixture flows, in other words the colloid-interface interactions.

The proposed approach allows us to underpin the role of thermodynamic processes in the discussed phenomena. In particular, we distinguish the physico-chemical interactions, which contribute to the equilibrium equation of state and allow us to separate the colloid-interface interactions from the interactions between the other components. Furthermore, we distinguish the irreversible phenomena, which exist only when part of the mixture experience relative motion.

In the proposed geometrical configuration, the main difference between the Marangoni flow and diffusio-osmosis is the direction of the flow. For Marangoni flow the mixture of a fluid and colloidal particles flows in the direction of the colloid concentration gradient, while for diffusio-osmosis the suspension flows in the direction opposite to the colloid concentration gradient. The model suggests that direction of the flow is controlled by the magnitude of the attractive part in the colloid-interface interaction potential. The latter is modelled in the context of the DLVO theory by the colloidinterface part of the pressure contribution to the total thermodynamic pressure. In particular, diffusio-osmosis is observed if the colloid-interface interactions are purely repulsive. In contrast, Marangoni flow is observed if the colloidinterface interaction potential has a large attractive term. At some magnitude of the attractive amplitude, the switching of the flow regime happens and we observe a sort of dynamic equilibrium that we called the neutral case: attractive and repulsive interactions neutralise each other and the net flow is close to the one obtained in absence of colloid-interface interactions (section 3.3).

The proposed universal description of diffusio-osmosis and Marangoni flow through a description of colloid-interface interactions may be extended further to include diffusiophoresis. When the interface is mobile in a stagnant fluid, by reciprocity, the interfacial driven transport induces the move of the interacting interface. This displacement is usually called diffusio-phoresis ${ }^{37-38}$. It is shown that the direction of the move in the colloid concentration gradient depends on the nature of the repulsion: the object goes toward low concentration in the case of colloid-interface repulsion and toward high concentration in the case of colloid-interface attraction. Overall, Fig. 10 summarizes how these four phenomena can be unified when considering the colloid-interface interactions.

It is worth noting that the direction of diffusio-phoresis depends on the direction of the colloid concentration gradient: the object will go in the direction of the high concentration of solute for which it feels attraction (or in the direction of low concentration of solute that it repels). This could have important implication for active or bio-colloids that could adjust their surface properties to move differently in concentration gradient.

The model can then help to progress in these investigations by giving the possibility to investigate the local effects of the interfacially driven transport. In real life case, the solute and the interface combine multiple interactions: they interacts both with repulsive (leading to osmotic like flow) and attractive contributions (leading to Marangoni like flow). Furthermore, active solute can also display patchy interactions on their surface that can also lead to specific transport properties ${ }^{39}$. The theoretical model developed here can help to solve this kind of complex interplay. A better understanding of diffusio-phoresis could also lead to design processes using diffusio-phoresis mechanisms ${ }^{40-41}$. The simulation could also be more realistic by integrating a dependence of the viscosity on the concentration and a non-ideality of the osmotic pressure to account for colloid-colloid interactions.

\section{Conclusions}

This paper serves several points. First, we have presented a model, which allows one to study Marangoni flow and diffusioosmosis as interfacial transport phenomena in a unified framework. Second, we have discovered a property, which is responsible for a change of the flow regime in these interfacial transport phenomena. Third, we have coupled the colloidinterface interactions with the interfacial transport phenomena. These flows are described as the consequence of the colloid-interface interaction that are repulsive in the case of osmosis (the semi-permeable membrane repels the colloids) or attractive for Marangoni flow (the interface attract the surfactant). This paper helps to progress in understanding the role played by physico-chemical properties (surface interactions) on chemical physics (transport phenomena).

From the thermodynamic perspective, the colloid-interface interactions are accounted by the particular equation of state. The modeling underlines how the colloid-interface interaction 1) plays a role on the mass and momentum balance 2 ) generates local transport phenomena (called interfacially driven transport) 3) changes the relative transport of an object and a fluid in a colloidal dispersion

The theoretical framework allows us to describe diffusioosmotic and Marangoni flows in the context of a unified approach. These flows are described as the consequence of the colloid-interface interaction that are repulsive in the case of osmosis (the semi-permeable membrane repels the colloids) or attractive for Marangoni flow (the interface attract the surfactant).

\section{Conflicts of interest}

There are no conflicts to declare.

\section{References}

1 J.S. Rowlinson, B. Widom, Molecular Theory of Capillarity. Clarendon Press, Oxford, 1982.

2 K.S. Glavatskiy, Multicomponent Interfacial Transport Described by the Square Gradient Model during Evaporation and Condensation, Springer, Berlin Heidelberg, 2011.

3 D. Bedeaux, Adv. Chem. Phys., 1986, 64, 47. 
4 K.S. Glavatskiy, J. Pharoah, S. Kjelstrup, J. Memb. Sci., 2013, 431, 96.

5 K.S. Glavatskiy, D. Bedeaux, J. Chem. Phys., 2010, 133, 144709.

6 K.S. Glavatskiy, D. Bedeaux, Phys. Rev. E, 2009, 79, 031608.

7 K.S. Glavatskiy, D. Bedeaux, Phys Rev E, 2008, 77, 061101. Erratum: Phys Rev E, 2011, 83, 019902.

8 J.D. Olarte-Plata, F. Bresme, Molecular physics, 2018, 116, 2032.

9 B.M. Law, S.P. McBride, J.Y. Wang, H.S. Wi, G. Paneru, S. Betelu, B. Ushijima, Y. Takata, B. Flanders, F. Bresme, H. Matsubara, T. Takiue, M. Aratono, Progress in surface science, 2017, 92, 1.

10 E. Ruckenstein, J. Colloid Interface Sci., 1981, 83, 77.

11 J. Lisenfeld, G.J. Grabovskij, C. Müller, J.H. Cole, G. Weiss, A.V. Ustinov, Nature Communications, 2015, 6, 6182.

12 S. Kjelstrup, L. de Meis, D. Bedeaux, J.-M. Simon, Eur. Biophys. J., $2008,38,59$.

13 H. Hoang, G. Galliero, J. Phys. Condens. Matter, 2013, 25, 485001.

14 J.-S. Bae, T.X. Nguyen, S.K. Bhatia, J. Phys. Chem. C, 2010, 114, 1046.

15 A. Torres-Carbajal, S. Herrera-Velarde, R. Castañeda-Priego, Phys. Chem. Chem. Phys., 2015, 17, 19557-19568.

16 S. Angioletti-Uberti, B. Mognetti, D. Frenkel, Phys. Chem. Chem. Phys., 2016, 18, 6373.

17 K. Nygård, Phys. Chem. Chem. Phys., 2017, 19, 23632.

18 Y. Hong, D. Velegol, N. Chaturvedi, A. Sen, Phys. Chem. Chem. Phys., 2010, 12, 1423.

19 P. Bacchin, J. Phys. Condens. Matter., 2018, 30, 294001.

20 P. Bacchin, Chem. Eng. Sci., 2017, 158, 208.

21 J.F. Morris, F. Boulay J. Rheol. 1999, 43, 1213.

22 P. Bacchin, Colloids and Surfaces A: Physicochemical and Engineering Aspects, 2017, 533, 147.

23 S. Marbach, H. Yoshida, L. Bocquet, J. of Chem Phys, 2017 146(19), 194701.

24 J.L. Anderson, Annu. Rev. Fluid Mech. 1989, 21, 61.

25 P.R. Nott, E. Guazzelli, O. Pouliquen, Phys. Fluids, 2011, 23, 043304.

26 J.E. Guyer, D. Wheeler, J.A. Warren, Comput. Sci. Eng., 2009, 11,6 .

27 J.L. Anderson, Annu. Rev. Fluid Mech., 1989 21, 61.

28 J.F. Brady, J. Fluid Mech. 2011, 667, 216.

29 C. Lee, C. Cottin-Bizonne, A.L. Biance, P. Joseph, L. Bocquet, C. Ybert, Phys. Rev. Lett. 2014, 112, 244501.

30 D. Velegol, A. Garg, R. Guha, A. Kar, M. Kumar, M.. Soft Matter, 2016, 12(21), 4686.

31 J.E. Drummond, J. E., M.I. Tahir, Int. J. Multiph. Flow, 1984, 10(5), 515.

32 D. Florea, S. Musa, J.M.R. Huyghe, H.M. Wyss, Proc. Natl. Acad. Sci., 2014, 111, 6554.

33 S. Lee, C. Boo, M. Elimelech, S. Hong, Journal of Membrane Science, 2010, 365(1-2), 34.

34 J.G. Kirkwood, F.P. Buff, J. Chem. Phys., 1949, 17, 338.

35 A. Dominguez, P. Malgaretti, M. N. Popescu, S. Dietrich, Phys. Rev. Lett. 2016, 116, 078301.

36 B. Cuenot, J. Magnaudet, B. Spennato, J. Fluid Mechanics, 1997, 339, 25.

37 L. Bocquet, E. Charlaix, Chem. Soc. Rev., 2010, 39 1073-95

38 P. Malgaretti, M.N. Popescu, S. Dietrich, Soft Matter, 2018, 14 1375.

39 J.T. Ault, P.B. Warren, S. Shin, H.A. Stone, Soft Matter, 2017 13, 9015 .

40 S. Shin, O. Shardt, P.B. Warren, H.A. Stone, Nat. Commun., 2017, 8, 15181.

41 E. Bianchi, R. Blaak, C.N. Likos, Phys. Chem. Chem. Phys., 2011, 13(14), 6397. 